\title{
COMPARATIVE APPLICATION OF METHODS FOR THE COMBINED LINES-NODES CAPACITY ASSESSMENT
}

\author{
ATIEH KIANINEJADOSHAH \& STEFANO RICCI \\ Sapienza University of Rome, Italy
}

\begin{abstract}
Nowadays, a considerable percentage of trains mature delays due to nodes and stations congestion. They are normally a combination of effects of routes conflicts in stations on lines and propagation in stations of delays suffered along the lines. It is a consequence of the variable values of capacity within the railway system caused by a combination of parameters, more relevant when short line sections separate nearby stations. Goal of the research is to compare some literature methods for the assessment of nodes and lines capacity to identify their reciprocal effects and innovative approaches. The work is included in a research framework with the final goal of traffic optimization and minimization of delays. In order to tackle the purpose, the paper introduces synthetically the methods and applies them systematically to a complex text network, including single and double track lines and various typologies of stations. The results of the methods applications are compared each other and with the outputs of a simulation approach. This analysis highlights the most appropriate application fields of single methods and the need of their integration and combination in terms of inputs, intermediate and final results. The review of single and coordinated use of these methods will move towards the definition of a comprehensive new methodological approach for combined lines-nodes capacity calculation. It will represent a powerful instrument to facilitate decision making on operational and infrastructural improvements of complex railway networks.
\end{abstract}

Keywords: capacity, circulation optimization, conflict, delays minimization, lines, stations.

\section{INTRODUCTION}

Capacity bottleneck area in railway networks are especially prone to generate delays due to the high number of trains' interactions, reason why they require special attention [1]. They are normally a combination of effects of routes conflicts in stations and lines and propagation in stations of delays suffered along the lines. On the other hand, the evolving European railway sector needs well managed and consolidated capacity calculation methods, for both lines and stations. It includes the limitations in the numerous interactions of the correlated factors, the complex structure of the railway system and the pre-existing conceptual and terminological variety [2]. These differences may lead to different capacity consumptions for stations and line sections and corresponding displacements of bottlenecks, which may lead to incorrect allocation of resources as well as to a reduced level of service on the railway network. In this context, the analysis and the identification of these bottlenecks will help to increase the capacity where necessary and to improve the corresponding passengers and freight services. The present paper introduces an approach to investigate the relationships between the capacity of lines and stations under disturbance conditions and hints for the potential synchronisation and generalisation of methods for a complex network.

Section 2 will deal with a synthetic bibliography of existing capacity methods. For computational purposes, quantitative reference application to a study network will exemplify figures, data, further assumptions and simplifications for the application of consolidated methodologies, e.g. Potthoff and UIC 406 methods. With the aim to calculate capacity and look for bottlenecks at network level, qualities and peculiarities of these methods move towards a quantitative integration in Section 3. Finally, Section 4 will summarise conclusions and identified perspective research developments. 


\section{STATE OF THE ART}

The evolution of research and the continuous increase of interest in railway capacity offer an extraordinarily rich bibliography of existing methodologies developed since 1950s. From [3] [4] [5] (first part of Table 1) arose 30 methods for station/node and line capacity assessment published until 2007.

More recently, additional contributions enriched the literature in the period 2011-2020 (second part of Table 1).

Table 1: Methods for station/node and line capacity assessment.

\begin{tabular}{|c|c|c|c|}
\hline Author & Year & Station/node & Line \\
\hline Muller & 1953 & $\checkmark$ & \\
\hline Bianchi & 1964 & & $\checkmark$ \\
\hline Bianchi & 1967 & & $\checkmark$ \\
\hline Formula FS & 1968 & & $\checkmark$ \\
\hline Potthoff & 1970 & $\checkmark$ & \\
\hline Formula DB & 1974 & & $\checkmark$ \\
\hline Petersen & 1974 & & $\checkmark$ \\
\hline Corazza, Florio & 1979 & $\checkmark$ & \\
\hline UIC 405-1 R & 1979 & & $\checkmark$ \\
\hline Cascetta, Nuzzolo & 1980 & & $\checkmark$ \\
\hline Corriere & 1982 & & $\checkmark$ \\
\hline UIC 405-2I & 1983 & & $\checkmark$ \\
\hline Florio, Malavasi & 1984 & $\checkmark$ & \\
\hline Corriere & 1984 & & $\checkmark$ \\
\hline Giuliani, Malavasi, Ricci & 1989 & $\checkmark$ & \\
\hline Chen, Harker & 1990 & & $\checkmark$ \\
\hline Corazza, Musso & 1991 & $\checkmark$ & \\
\hline Canciani & 1991 & & $\checkmark$ \\
\hline Malaspina, Reitani & 1995 & & $\checkmark$ \\
\hline Galaverna, Sciutto & 1999 & $\checkmark$ & $\checkmark$ \\
\hline Galatola & 2002 & $\checkmark$ & $\checkmark$ \\
\hline Delfino, Galaverna & 2003 & & $\checkmark$ \\
\hline Formula RFI & 2004 & & $\checkmark$ \\
\hline UIC 406-R & 2004 & & $\checkmark$ \\
\hline Galatola & 2004 & $\checkmark$ & $\checkmark$ \\
\hline Galatola & 2005 & $\checkmark$ & $\checkmark$ \\
\hline Genovesi, Ronzino & 2006 & & $\checkmark$ \\
\hline Burdett, Kozan & 2006 & & $\checkmark$ \\
\hline Ciuffini & 2007 & $\checkmark$ & \\
\hline
\end{tabular}


Table 1: (Continued)

\begin{tabular}{llll}
\hline Author & Year & Station/node & Line \\
\hline Kaas, Landex & 2007 & & $\checkmark$ \\
Landex [6] & 2011 & $\checkmark$ & \\
Ricci, Dicembre [7] & 2011 & & $\checkmark$ \\
Heydar, Petering, Bergmann [8] & 2013 & & $\checkmark$ \\
Goverde, Corman, D'Ariano [9] & 2013 & & \\
Ricci, Malavasi, Molková, Rotoli [10] & 2014 & $\checkmark$ & $\checkmark$ \\
Zhang [11] & 2015 & & $\checkmark$ \\
Zhang, Nie [12] & 2016 & & $\checkmark$ \\
Lai, Ip [13] & 2017 & & $\checkmark$ \\
Jamili [14] & 2018 & & $\checkmark$ \\
Yan, Goverde [15] & 2019 & & \\
Wang, Nie, Tan [16] & 2020 & & \\
\hline
\end{tabular}

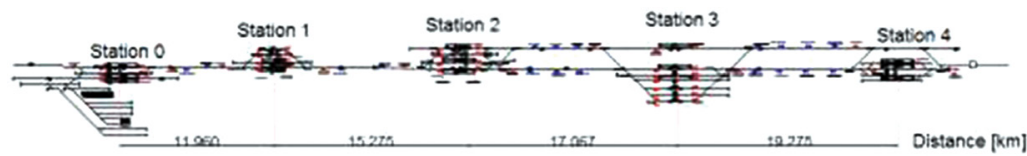

Figure 1: Case study network layout.

\section{METHODOLOGIES APPLICATION TO AN INTEGRATED CASE STUDY}

The value of capacity over a railway network is variable according to the effects of various parameters. The ongoing research deals with the investigation of this variability with a special focus on the interactions between lines and stations features, hardly considered in the most consolidated models and methodologies.

In view of the setup of a synthetic method, it is necessary to quantify the effects of the parameters above on the capacity, here tested on a reference network layout, including three stations, two single track and two double track line sections (Fig. 1).

\subsection{Operation at the stations}

Despite the normally larger amount of tracks, stations and larger nodes may represent capacity bottlenecks of the networks [17] when their morphology reduces the compatibility of the movements. Moreover, this effect is depending on the frequency of use of the station routes, which share sections with the nearby lines. The Potthoff method [18] includes combinatorial procedures able to quantify the utilisation rate of single routes, station areas and the station as a whole. Figure 2 shows the architecture of the automatic calculation procedure setup for it.

This method assumes that trains could arrive at any instant with the same probability within the reference time $T$; therefore, it does not require an assigned timetable, which simplifies its application [19] [20]. Moreover, the application considered the following hypotheses: 
1. The single-track line has one block and one warning signal per direction;

2. All stations tracks are bidirectional;

3. The release assumptions are:

a. Train entrance to station: movement starting from last warning signal with line speed $(100 \mathrm{~km} / \mathrm{h})$ and releases the line for the second train by passing beyond the conflict point with the deviation speed $(60 \mathrm{~km} / \mathrm{h}$ ) and stop (length of train counted once after the conflicting point);

b. Train exit form station: accelerated from the departure signal to reach the deviation speed $(60 \mathrm{~km} / \mathrm{h})$ and, after the conflict point, it will increase the speed up to the maximum speed $(100 \mathrm{~km} / \mathrm{h})$ until the conflicting point of next station;

4. First in, first out (FIFO) service discipline for the station;

5. Constant probability density for the arrival of trains during the reference period $T$.

The following parameters are also considered:

- $N=\Sigma n_{i}=\Sigma n_{j}=$ total number of movements; $n_{\text {med }}=$ average number of simultaneous movements allowed by the layout; $t_{\text {med }}=$ average occupation time of routes.

The described approach bases on a combination of train paths. Therefore, the method allows calculating the total delay $\left(\Sigma R_{i j}\right)$ generated in the node as the sum of the delays generated by each incompatibility between two routes $i$ and $j$. The ratio between $\Sigma R_{i j}$ and $n_{\text {med }}$

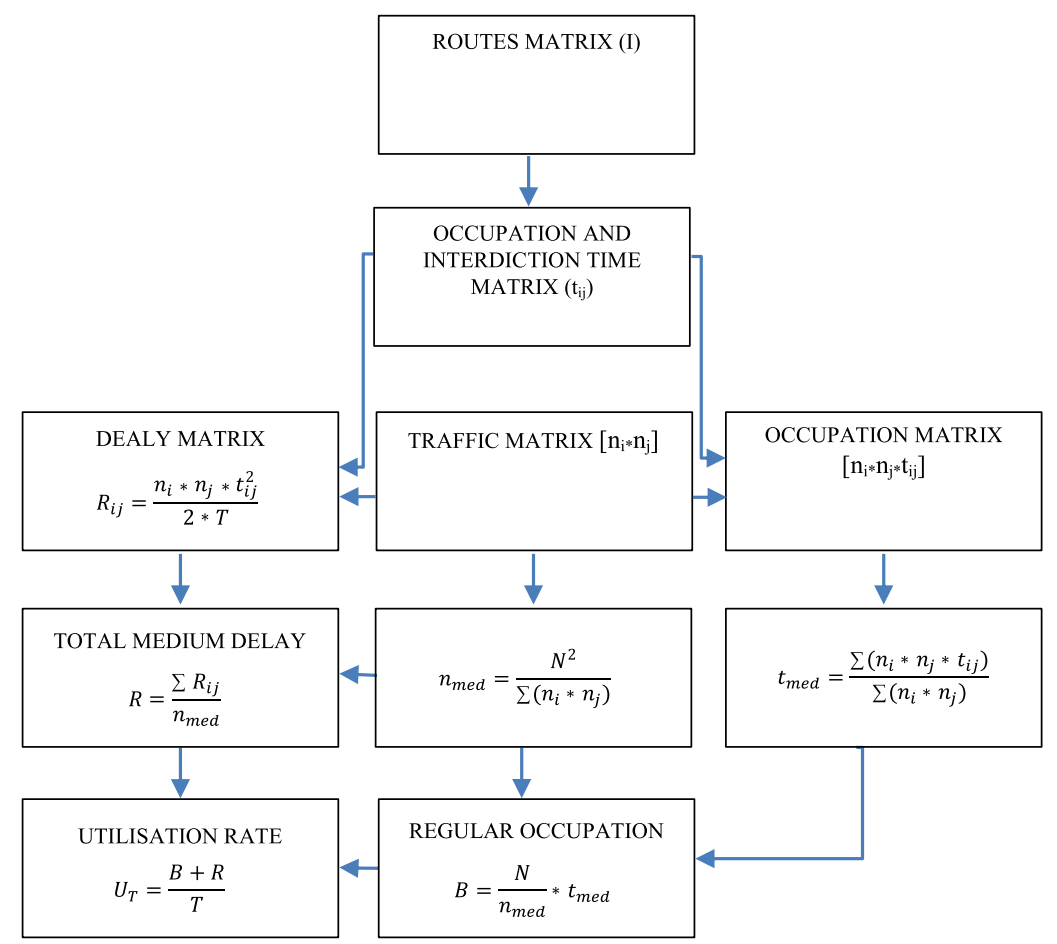

Figure 2: Potthoff method procedure. 
represents the total delay obtained considering movements of $n_{\text {med }}$ trains simultaneously as well as the average delay/train is the following:

$$
R_{\text {med }}=\frac{\sum R_{i j}}{N}
$$

As an example, with reference to the stations included in the case study network, the diagram in Fig. 3 shows the average delay/train generated by a variable amount of running trains.

Moreover, the global utilization rate $U_{t}$ is determined by:

$$
U_{t}=\frac{\left(\frac{N}{n_{\text {med }}} * t_{\text {med }}\right)+\left(\frac{\sum R_{i j}}{n_{\text {med }}}\right)}{T}=\frac{B+R}{T}
$$

The results of the calculation of the global utilisation rates corresponding to the increase of traffic are in the diagrams reported in Fig. 4.

The calculation of the corresponding capacity needs to fix a reference value of punctuality, e.g. in terms of maximum allowed delay, and to derive from it the corresponding maximum amount of trains compatible with such value.

In respect of the specific purpose to evaluate the capacity as an integrated concept across stations and line sections, this calculation will follow the next paragraph.

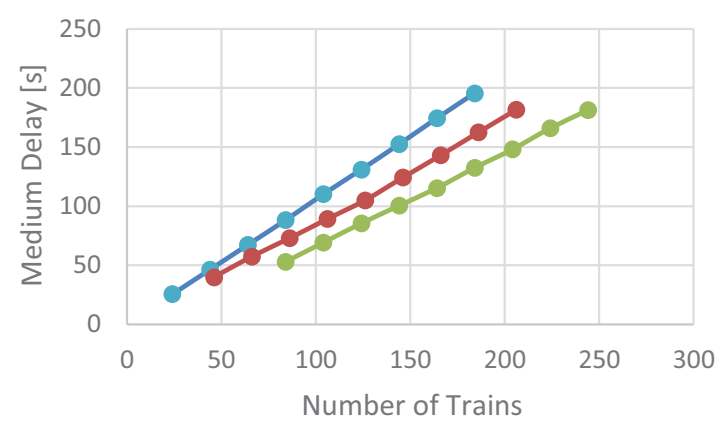

Figure 3: Average delay/train generated in stations 1 (blue), 2 (red) and 3 (green).

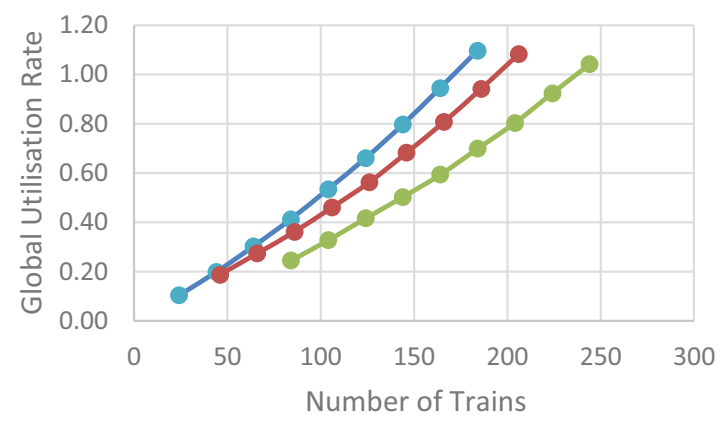

Figure 4: Global utilisation rate calculated for stations 1 (blue), 2 (red) and 3 (green). 
3.2 Operation along the line sections

The UIC 406 capacity method is an easy and effective way to evaluate the capacity and its utilisation along railway lines [21]. From presentations, elaborations and discussions of this method in a number of papers [22] [23] [24] emerges that capacity consumption on railway lines depends on both the infrastructure asset and the timetable.

Timetables planning is normally for the entire railway network, which means that the specific timetable in the concerned area depends upon those outside it, in a typical network effect [25]. Figure 5 shows the architecture of the automatic procedure developed to apply UIC 406 method.

The simplified exemplificative calculation bases on the following assumptions:

- All trains are similar as for dimensions and performances;

- $t_{f m}$ corresponds:

- On single track line to time needed from departure signals of two consecutive stations;

- On double track line to headway for two consecutive sections in the same direction.</

The capacity utilisation of a timetable on a specific infrastructure is basing on the following consumption of times:

$$
k=t_{f m}+t_{r}+t_{z u}+D
$$

- $t_{f m}=$ infrastructure occupation; $t_{r}=$ buffer time; $t_{z u}=$ supplement for multiple sections on double track lines; $D=$ supplement reserved for maintenance, here considered in the daily time exceeding the reference time $T=18 \mathrm{~h}$.

The infrastructure occupation $\left(t_{f m}\right)$ is the accumulation of the consumed time evaluated by doing the timetable compression. The buffer time $\left(t_{r}\right)$ is between train paths to reduce the transfer of delays from one train to another one. The supplements $\left(t_{z u}\right.$ and $\left.D\right)$ are further virtual infrastructure occupation.

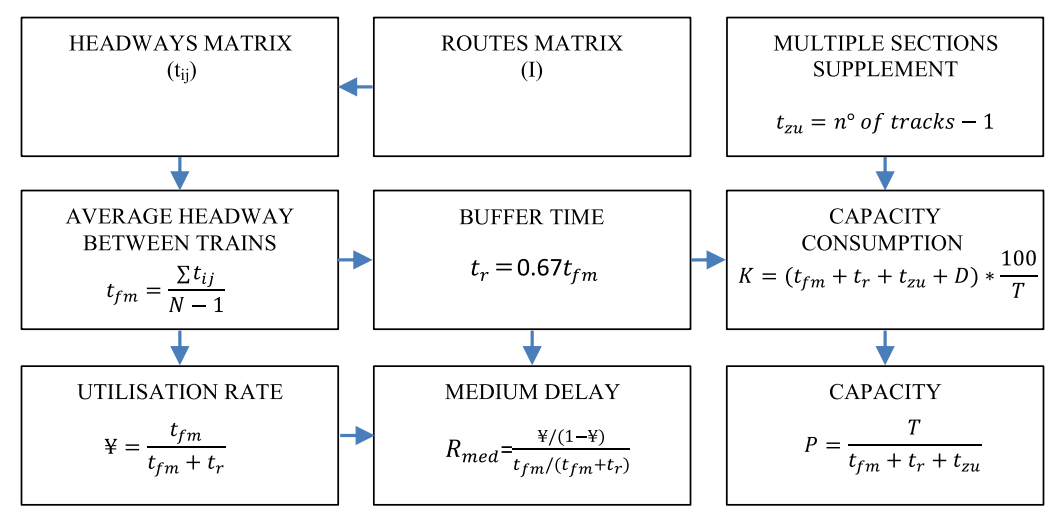

Figure 5: UIC 406 method procedure. 
The percentage of capacity consumption $K$ is the quotient of total consumed times and the reference time $T$.

$$
K=k *\left(\frac{100}{T}\right)
$$

The capacity consumption represents the chained occupancy rate, as the compression is inevitably not possible for a partition consisting of one specific block interval only. Instead of that, an examination partition can consist of more than one block interval [26].

\section{CAPACITY RESULTS}

In our research, we are investigating the mutual effect of lines and stations features on capacity. Therefore, though the corresponding methods, separately conceived and applied, provide their own values, their input-output integrated use is basing on the common standard of punctuality (threshold of delay).

The presented approach is basing on the punctuality indicators calculated according to the UIC 406 methods. The standard level of punctuality is that corresponding to $t_{r}=0.67 t_{f m}$, normally applied for calculation of daily capacity of lines.

The most expressive punctuality indicator, selected as an example of common parameter across lines and stations methods, is the average delay/train, calculated as:

$$
R_{\text {med }}=\frac{¥ /(1-¥)}{t_{f m} /\left(t_{f m}+t_{r}\right)}
$$

- $¥=$ utilisation rate; $t_{f m}=$ average headway between trains; $t_{r}=$ buffer time.

According to the proposed approach, the corresponding numerical value $R_{\text {med }}=136 \mathrm{~s}$, used for the calculation of the capacity for single track and double track section, become an input for the station capacity calculation by using the results showed in Fig. 3 entered as input by the value above of the average delay/train.

Therefore, the corresponding values of capacity for the stations are deriving from Fig. 3.

In the meantime, Fig. 6 is representing, in a global overview, the capacity calculated with reference to the common level of punctuality expressed by the average delay/train of $136 \mathrm{~s}$.

All trains are in transit, except those finish their ride in the intermediate stations, reflected by used capacity.

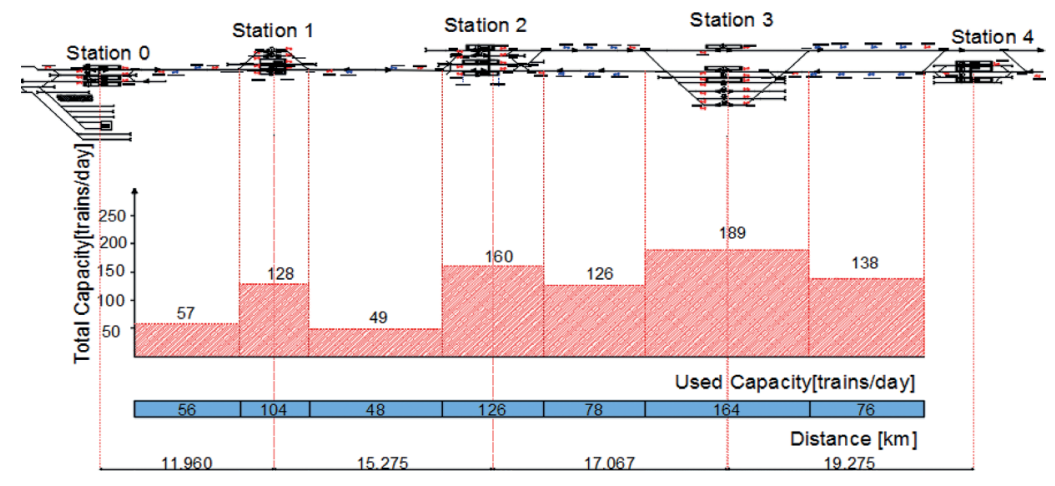

Figure 6: Calculated and used capacity of lines and stations. 


\section{CLOSING REMARKS}

The paper fixed the pillars for a comprehensive new methodological approach to combine lines and nodes capacity. The achievements resulting from the present investigations arose from an integrated application of different literature methods for the assessment of nodes and lines capacity on a test network. The link between the methods is the common expected delay level. The work is included in a research framework with the final goal of traffic optimization and minimization of delays generated within complex railway networks

The next planned development is the identification of parameters affecting the capacity of both lines and nodes and the quantification of these effects by sensibility analysis on the identified fundamental parameters.

The focus of first ongoing investigations will be in the direction of the effects on the capacity of the incompatibilities between the routes on the opposite sides of stations.

Moreover, the validation of the methodological approaches will include:

- Traffic simulation on the concerned railway networks:

- Crosscheck of achieved results with collected real operation data.

\section{REFERENCES}

[1] Lüthi, M., Medeossi, G. \& Nash A., Evaluation of an integrated real-time rescheduling and train control system for heavily used areas. RailHannover - International Seminar on Railway Operations Modelling and Analysis. Hannover, Germany, 2007.

[2] Kontaxi E., Ricci S. (2012). Railway capacity handbook: a systematic approach to methodologies. Procedia - Social and Behavioral Sciences, 48, 2012.

[3] Kontaxi E., Ricci S. (2009). Techniques and methodologies for carrying capacity evaluation: comparative analysis and integration perspectives. Ingegneria Ferroviaria, 64(12), 2009.

[4] Kontaxi E., Ricci S. (2011). Calculation of railway network capacity: comparing methodologies for lines and nodes. RailRome - International Seminar on Railway Operations Modelling and Analysis. Rome, Italy, 2011.

[5] Kontaxi E., Ricci, S. (2010). Railway capacity analysis: methodological framework and harmonization perspectives. WCTR - World Conference on Transportation Research. Lisboa, Portugal, 2010.

[6] Landex A. (2011). Capacity at railway stations. World Congress on Railway Research WCRR 2011. Lille, France.

[7] Dicembre A., Ricci S. (2011). Railway traffic on high density urban corridors: capacity, signalling and timetable. Journal of Rail Transport Planning \& Management, 1(2).

[8] Heydar M., Petering M.E., Bergmann D.R. (2013). Mixed integer programming for minimizing the period of a cyclic railway timetable for a single track with two train types. Computers \& Industrial Engineering, 66(1).

[9] Goverde R M., Corman F., D'Ariano A. (2013). Railway line capacity consumption of different railway signalling systems under scheduled and disturbed conditions. Journal of Rail Transport Planning \& Management, 3(3).

[10] Malavasi G., Molková T., Ricci S., Rotoli, F. (2014). A synthetic approach to the evaluation of the carrying capacity of complex railway nodes. Journal of Rail Transport Planning and Management, 4(1-2). 
[11] Zhang J. (2015). Analysis on-line capacity usage for China high speed railway with optimization approach. Transportation Research Part A: Policy and Practice, 77.

[12] Zhang X., Nie L. (2016). Integrating capacity analysis with high-speed railway timetabling: a minimum cycle time calculation model with flexible overtaking constraints and intelligent enumeration. Transportation Research Part C: Emerging Technologies, 68.

[13] Lai Y. C., Ip C. S. (2017). An integrated framework for assessing service efficiency and stability of rail transit systems. Transportation Research Part C: Emerging Technologies, 79 .

[14] Jamili A. (2018). Computation of practical capacity in single-track railway lines based on computing the minimum buffer times. Journal of Rail Transport Planning \& Management, 8(2), 91-102.

[14] Yan, F., \& Goverde, R. M. (2019). Combined line planning and train timetabling for strongly heterogeneous railway lines with direct connections. Transportation Research Part B: Methodological, 127.

[15] Wang R., Nie L., Tan Y. (2020). Evaluating Line Capacity with an Analytical UIC Code 406 Compression Method and Blocking Time Stairway. Energies, 13(7), 1853.

[16] Armstrong J., Preston J. (2017). Capacity utilisation and performance at railway stations. Journal of Rail Transport Planning and Management, 7(3).

[17] Potthoff G. (1965). Verkehrsströmungslehre. Transveb, Berlin, Germany, 1965.

[18] Rotoli F., Malavasi G., Ricci S. (2016). Complex railway systems: capacity and utilisation of interconnected networks. European Transport Research Review, 8(4).

[19] Malavasi G., Rotoli, F. (2008). Carrying capacity of complex railway nodes. AATT 2008 - International Conference on Applications of Advanced Technologies in Transportation, Athens, Greece.

[20] UIC - International Union of Railways (2004). Leaflet 406, Capacity. Paris, France, 2004.

[21] Wahlborg M. (2004). Banverket experience of capacity calculations according to the UIC capacity leaflet. WIT Transactions on the Built Environment, 74, 2004.

[22] Prinz R., Höllmüller J. (2005). Implementation of the UIC 406 capacity calculation method at Austrian railways (ÖBB). WCRR 2006 - World Conference on Railway Research. Montreal, Canada.

[23] Landex A., Kaas A.H., Schittenhelm B., Schneider-Tilli, J. (2006). Evaluation of railway capacity. Trafficdays, Aalborg, Denmark, 2006.

[24] Hansen S., Landex A., Kaas A. H. (2006). The network effects of railway investments. International conference WIT Transactions on the Built Environment, 74, 2006.

[25] Linder T. (2011). Applicability of the analytical UIC Code 406 compression method for evaluating line and station capacity. Journal of Rail Transport Planning \& Management, 1(1), 2011. 